\title{
MATHEMATICAL MODEL FOR BIRD FLU DISEASE TRANSMISSION WITH NO BIRD MIGRATION
}

S. C. INYAMA

(Received 2, August 2007; Revision Accepted 22, July 2008)

\begin{abstract}
In this paper a mathematical model for the transmission dynamics of bird flu among birds and humans is presented. The model assumes that there is no migration of birds in the susceptible bird population immediately the disease starts. The model formulated is analyzed using dynamical systems theory. The analysis of the steady state and its stability show that the system will be stable if there is a bound on the growth (birth) of birds in the community $\left(\alpha_{B}\right)$. This means that the disease will die out after enough time if there is a bound on the growth rate of birds. We also looked at the endemic flu state and showed that the disease will persist if there is a bound on the infection transition rate from birds to birds $\left(\beta_{\mathrm{B}}\right)$.
\end{abstract}

KEY WORDS: Mathematical model, bird - flu disease, transmission, steady state, stability.

\section{INTRODUCTION}

Bird flu also called avian influenza is a major contributor to mortality and morbidity through out the world [4]. It is a respiratory infection in humans and birds caused by an RNA virus in the family of orthomyxoviridae. According to World Health Organization respiratory infections, (including bird flu) killed an estimated over four million people in 1999, but it is very difficult to calculate how mortality is caused by flu $[1,9]$.

Bird flu is majorly spread by migratory birds, as they move around the world to seasonal breeding and feeding grounds, infect domestic flocks (birds) around the world. Since December 2003, bird flu has killed or culled more then 150 million birds in the world. Also 63 out of 124 infected humans have died since December 2003 [9].

The flu appeared in Hong Kong in 1997 and since then, highly pathogenic H5N1 avian flu has spread to 15 countries in Asia, Europe and now in Africa including Nigeria [8].

However, there is so far no outbreak of human pandemic influenza anywhere in the world today, though the signs indicate that it may happen [8]. Bird flu has a lot of economic impact in the whole world and it is estimated that this has already exceeded 10 billion dollars [8].

Some mathematical models have been developed to help understand the transmission dynamics of bird flu. A computer model was developed by a team of United Kingdom and United States of America researchers. The model predicts that "if a bird flu pandemic breaks out, only a vaccine-one better than any existing flu vaccine-can keep it from infecting virtually everyone in the United States of America and the rest of the world. But the model offers hope, too. A range of public health strategies and luck might soften the blow". The computer model is the brainchild of researchers including Ferguson and Burke [5].

Baym, [2] presented a paper which discussed the basics of mathematical epidemiology of bird flu. It focused on differential models but also touched on time series and spatial model. Longini, et. al. [6] developed mathematical models of flu outbreak. The models predict exactly how far a flu outbreak will spread under different circumstances. In the model they made a prediction, "if a flu outbreak were like the relatively mild 1975 Asian flu virus, it would infect 93 million Americans and cause 164,000 United States of America deaths".

S. C. Inyama, Department of Mathematics and Computer Science, Federal University of Technology, Owerri, Imo State. 
Yusuf and Okosun [10] developed a mathematical model to examine the population dynamics with respect to the disease and its transmission. The appropriate systems of ordinary differential equations formulated were solved numerically and the results were analyzed. The result showed that the spread of the virus will continue as long as there are infected birds and there is tendency of human infection sooner or later. Their model assumed that there is a migration into the susceptible class.

\section{Model Formulation}

We present an S -I- R compartmental model which describes the dynamics of the bird flu disease in bird (chicken) and human populations. In humans, the disease causes no permanent immunity, though there exist some kind of temporary immunity [4]. The recovered humans join the susceptible class and then get exposed again. The recovered category for birds is ignored since birds (chickens) hardly recover from highly pathogenic avian influenza [7].

\section{Symbols and Parameters}

Below are the symbols and parameters used in this model.

$\mathrm{N}_{\mathrm{B}}=$ Total number of birds in the location of interest

$\mathrm{N}_{\mathrm{H}}=$ Total number of humans in the location of interest

$\alpha_{B}=$ Average birth rate of birds

$\alpha_{H}=$ Average transmission rate from birds to humans

$\beta_{H}=$ Infection transmission rate from birds to humans

$\beta_{\mathrm{B}}=$ Infection transmission rate from birds to birds

$\delta_{\mathrm{H}}=$ Natural death rate in humans

$\delta_{\mathrm{B}}=$ Natural death rate in birds

$\lambda_{\mathrm{H}}=$ Flu-induced death rate in humans

$\lambda_{\mathrm{B}}=$ Flue - induced death rate in birds

$r \quad=$ Recovery rate for humans (per day)

$\mathrm{S}_{\mathrm{H}}(\mathrm{t})=$ Susceptible humans at time $\mathrm{t}$

$I_{H}(t)=$ Infectious Humans at time $t$.

$\mathrm{I}_{\mathrm{B}}(\mathrm{t})=$ Infectious birds at time $\mathrm{t}$.

$\mathrm{S}_{\mathrm{B}}=$ Susceptible birds at time $\mathrm{t}$.

It is important to observe that

$$
\begin{aligned}
& N_{H}=S_{H}+I_{H} \\
& N_{B}=S_{B}+I_{B}
\end{aligned}
$$

\section{Schematic description of the model}

Below is the schematic description of the proposed model. This makes it easier to understand the model equations to be presented later.

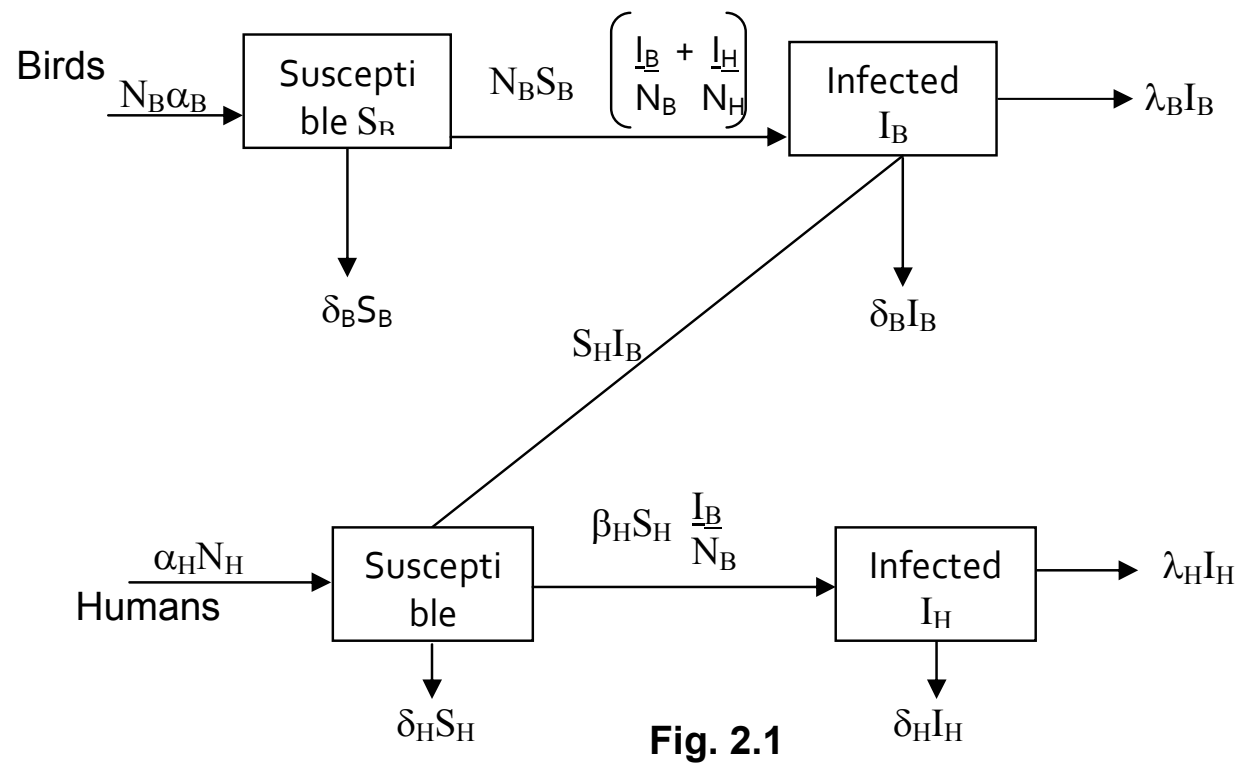


Assumptions of the model

Below are the assumptions of the model:

1. There is no migration into the susceptible class of the birds immediately the disease starts in a community.

2. There is no infection between human and human.

3. There is no recovery in the infected birds [9].

\subsection{The Model}

From the above diagram, we can now write the mathematical model for the birds and humans as an ordinary differential equation.

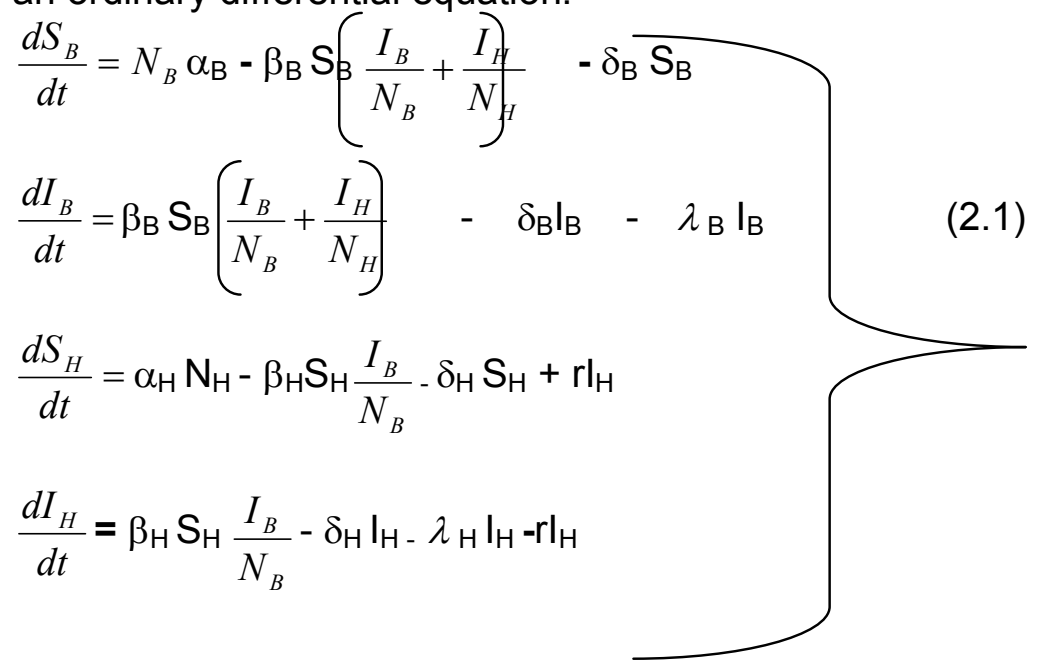

Equation (2.1) can be explained thus. The first equation indicates that the change in the number of susceptible birds with change in time will be the number of birds born less the number of susceptibles that have been infected by both birds and humans, less the number of susceptibles that died as a result of non-flu causes.

The second equation says that the number of infected birds with change in time is the number of birds infected by both birds and humans, less the number of birds that have died as a result of the flu and as a result of natural death.

The third equation indicates that the change in the number of human susceptibles with change in time, is the number of humans born and the number of recovered infected humans less the number that died as a result of non-flu causes. The last equation says that the change in the number of inflected humans with change in time is the number of infected humans less those who recovered and those who died as a result of both the flu and natural death.

\section{Steady State and its Stability}

The disease-free steady state is obtained by setting.

$\underline{\mathrm{dS}} \underline{\mathrm{B}}_{\mathrm{B}}=\underline{\mathrm{dl}} \underline{\mathrm{B}}=\underline{\mathrm{dS}} \underline{\mathrm{S}}_{\underline{\mathrm{H}}}=\underline{\mathrm{dl}}_{\underline{\mathrm{H}}}=0$

in equation (2.1) and taking all the infected terms to be zero. This gives

$$
\mathrm{E}_{\mathrm{o}}=\left(S_{B}^{o}, I_{B}^{o}, S_{H}^{O}, I_{H}^{O}\right)=\underset{\delta_{\mathrm{B}}}{\left(\underline{\mathrm{N}}_{\mathrm{B}} \underline{\alpha}_{\mathrm{B}}, 0, \underline{\mathrm{N}}_{\underline{\mathrm{H}}} \underline{\alpha}_{\underline{H}}, 0\right)} \delta_{\mathrm{B}}
$$


Theorem 3.1 [3]

The zero equilibrium of $\frac{\mathrm{du}}{\mathrm{dt}}=\mathrm{AU}(\mathrm{t})$ is globally asymptotically stable if and only if the real parts of the eigenvalues of $A$ are negative and unstable otherwise.

Linearizing (2.1) we obtain the Jacobian of (2.1) as

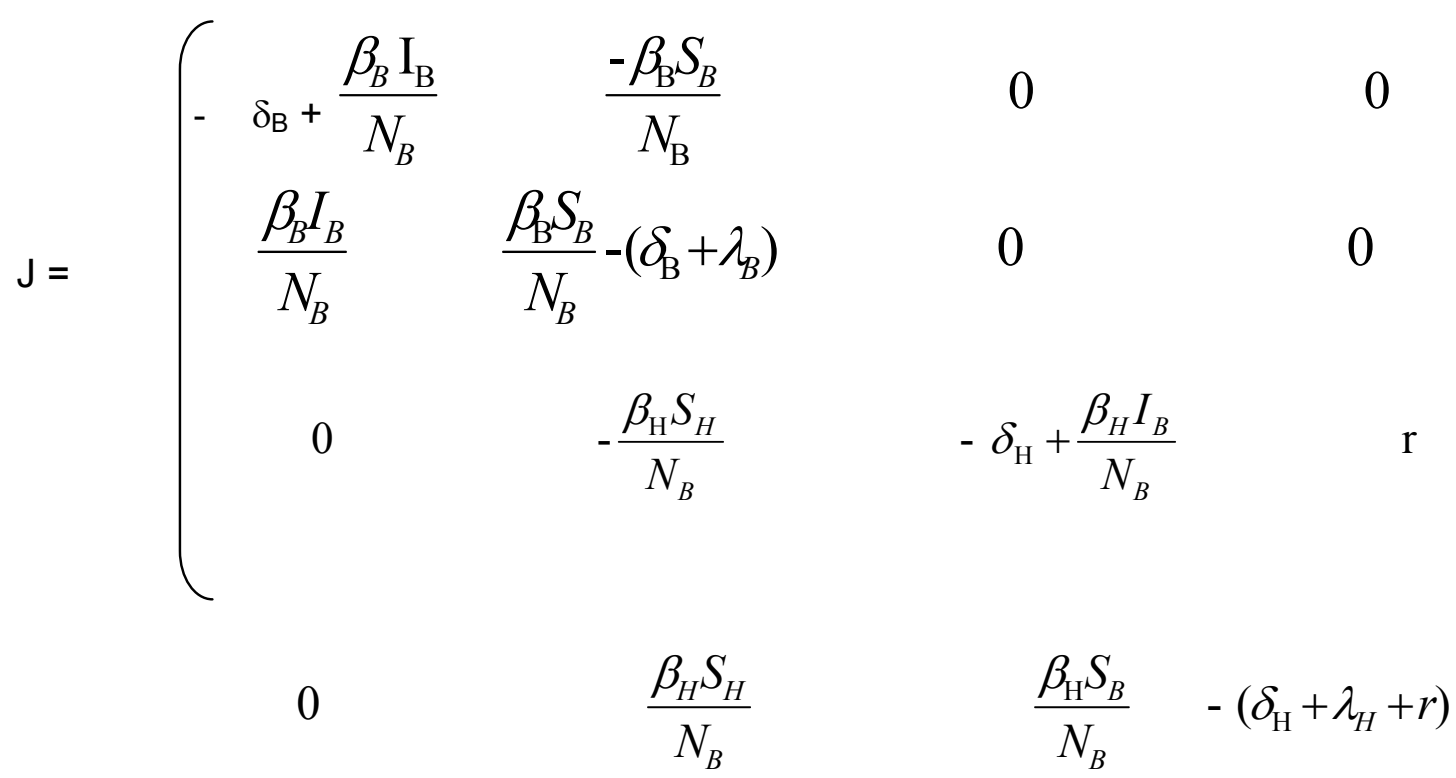

Evaluating $\mathrm{J}$ at $\mathrm{E}_{\mathrm{o}}$ yields
$\left\{\begin{array}{rrr} & & -\delta_{B} \\ & 0 & \\ \mathrm{~J}_{0}= & & \\ & & \\ & & \\ & & \\ & & \end{array}\right.$

$$
\begin{array}{r}
-\frac{\alpha_{\mathrm{B}} \beta_{B}}{\delta_{B}} \\
\frac{\beta_{\mathrm{B}} \alpha_{B}}{\delta_{B}}-\left(\delta_{\mathrm{B}}+\lambda_{B}\right) \\
-\frac{\beta_{\mathrm{H}} \alpha_{H} N_{H}}{N_{B} \delta_{H}} \\
\frac{\beta_{H} \alpha_{H} N_{H}}{N_{B} \delta_{H}}
\end{array}
$$$$
0
$$
0
$-\delta_{\mathrm{H}}$
r
$-\left(\delta_{\mathrm{H}}+\lambda_{H}+r\right)$

The eigenvalues of J0 are

$-\delta_{\mathrm{B}}, \frac{\beta_{\mathrm{B}} \alpha_{B}}{\delta_{B}}-\left(\delta_{\mathrm{B}}+\lambda_{B}\right),-\delta_{\mathrm{H}},-\left(\delta_{\mathrm{H}}+\lambda_{H}+r\right)$

If

$$
\frac{\beta_{B} \alpha{ }_{B}}{\delta_{B}}-\left(\delta_{\mathrm{B}}+\lambda_{B}\right)<0
$$


Then all the eigenvalues will be negative. This means that the disease-free steady state is

stable. (3.4) implies that

$$
\begin{aligned}
& \frac{\beta_{B} \alpha_{B}}{\delta_{B}}<\delta_{\mathrm{B}}+\lambda_{B} \\
& \Rightarrow \alpha_{B}<\frac{\left(\delta_{\mathrm{B}}+\lambda_{B}\right) \delta_{B}}{\beta_{B}}
\end{aligned}
$$

(3.5) tells us that there should be a bound on the growth (birth) of the birds in the community. One (3.5) is satisfied, then the disease dies out after enough time has passed and then we achieve a disease (flu) -free situation. That is, the disease-free state is asymptotically stable.

\subsection{Endemic Flu}

A gain setting the derivatives in (2.1) to zero and solving algebraically, we obtain the endemic steady state.

Observe that,

$$
\begin{gathered}
\mathrm{S}^{0}=\mathrm{N}-\mathrm{I}^{0} \\
I_{B}^{0}=\mathrm{N}_{\mathrm{B}} \frac{\left(\alpha_{\mathrm{B}}-\delta_{\mathrm{B}}\right)}{\lambda_{B}} \\
I_{H}^{0}=\mathrm{N}_{\mathrm{H}} \frac{\left(\alpha_{\mathrm{H}}-\delta_{\mathrm{H}}\right)}{\lambda_{H}}
\end{gathered}
$$

Hence,

Hence the endemic steady state is

$$
\begin{aligned}
& \left(S_{B}^{O}, I_{B}^{O}, S_{H}^{O}, I_{H}^{O}\right)=\left(\mathrm{N}_{\mathrm{B}}-\mathrm{I}_{\mathrm{B}}^{\mathrm{O}}, I_{B}^{O}, N_{H}-\mathrm{I}_{\mathrm{H}}^{\mathrm{O}}, I_{H}^{O}\right) \\
& \left.=\frac{N_{B}}{\lambda_{B}}\left(N_{B}-\alpha_{\mathrm{B}}-\delta_{\mathrm{B}}\right) \frac{N_{B}}{\lambda_{B}}\left(\alpha_{B}-\delta_{\mathrm{B}}\right) \frac{N_{H}}{\lambda_{H}}\left(\lambda_{H}-\alpha_{\mathrm{H}}-\delta_{\mathrm{H}}\right), \frac{N_{H}}{\lambda_{H}}\left(\alpha_{H}\right]_{H}\right)
\end{aligned}
$$

The Jacobian then becomes 


$$
\begin{aligned}
& \text { - }\left[\begin{array}{llll}
\left.\delta_{\mathrm{B}}+\frac{\beta_{B}}{\lambda_{B}}\left(\alpha_{B}-\delta_{\mathrm{B}}\right)\right] & -\frac{\beta_{\mathrm{B}}}{\lambda_{\mathrm{B}}}\left(\lambda_{B}-\alpha_{\mathrm{B}}-\delta_{\mathrm{B}}\right) & 0 & 0
\end{array}\right. \\
& \frac{\beta_{B}}{\lambda_{B}}\left(\alpha_{B}-\delta_{\mathrm{B}}\right) \quad \frac{\beta_{\mathrm{B}}}{\lambda_{\mathrm{B}}}\left(\lambda_{B}-\alpha_{\mathrm{B}}-\delta_{\mathrm{B}}\right)-\left(\delta_{\mathrm{B}}+\lambda_{B}\right) \quad 0 \quad 0 \\
& \left.\begin{array}{cccc}
\mathrm{J}_{\mathrm{E}}= & -\frac{\beta_{B} N_{H}}{N_{B}}\left(\lambda_{H}-\alpha_{\mathrm{H}}-\delta_{\mathrm{H}}\right) & -\left[\delta_{\mathrm{H}}+\frac{\beta_{H}}{\lambda_{B}}\left(\alpha_{B}-\delta_{\mathrm{B}}\right)\right] & \mathrm{r} \\
0 & \frac{\beta_{\mathrm{B}} N_{H}}{N_{B}}\left(\lambda_{H}-\alpha_{\mathrm{H}}-\delta_{\mathrm{H}}\right) & \frac{\beta_{\mathrm{H}}}{\lambda_{\mathrm{B}}}\left(\alpha_{B}-\delta_{\mathrm{B}}\right) & -\left(\delta_{\mathrm{H}}+\lambda_{H}+r\right)
\end{array}\right)
\end{aligned}
$$

In a pure endemic case, $r$ is zero since there may be no recovery (which means the non-existence of susceptibles). Hence $J_{E}$ becomes.

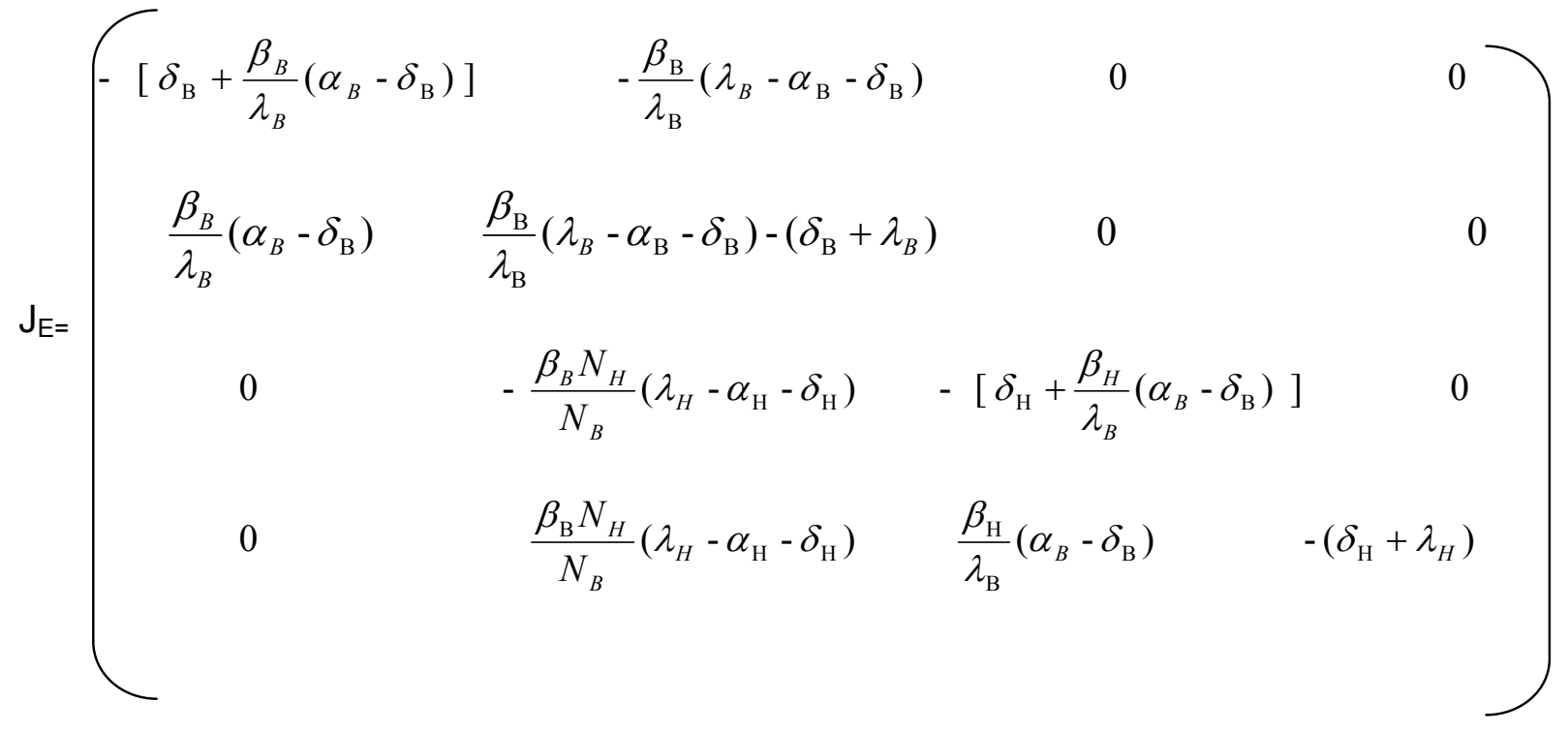

The eigenvalues of $J_{E}$ are

$$
\begin{aligned}
& -\left[\delta_{\mathrm{B}}+\frac{\beta_{B}}{\lambda_{B}}\left(\alpha_{B}-\delta_{\mathrm{B}}\right)\right], \frac{\beta_{\mathrm{B}}}{\lambda_{\mathrm{B}}}\left(\lambda_{B}-\alpha_{\mathrm{B}}-\delta_{\mathrm{B}}\right)-\left(\delta_{\mathrm{B}}+\lambda_{B}\right),-\left[\delta_{\mathrm{H}}+\frac{\beta_{H}}{\lambda_{B}}\left(\alpha_{B}-\delta_{\mathrm{B}}\right)\right], \\
& -\left(\delta_{\mathrm{H}}+\lambda_{H}\right)
\end{aligned}
$$

By Theorem 3.1 above, the endemic flu state will be asymptotically stable only if

$$
\begin{aligned}
& \frac{\beta_{B}}{\lambda_{B}}\left(\lambda_{B}-\alpha_{\mathrm{B}}-\delta_{\mathrm{B}}\right)-\left(\delta_{\mathrm{B}}+\lambda_{B}\right)<0 \\
& \Rightarrow \beta_{B}<\frac{\lambda_{B}\left(\delta_{B}+\lambda_{B}\right)}{\lambda_{B}-\alpha_{\mathrm{B}}-\delta_{\mathrm{B}}}
\end{aligned}
$$


Expression (3.9) means that the disease will persist (will be endemic) if the infection transmission rate from birds to birds has a bound. This means that the infection will be more among humans.

\section{SUMMARY, CONCLUSION AND RECOMMENDATION}

In this paper we have been able to develop a mathematical model for the transmission dynamics of bird flu among birds and humans. The model assumed that the susceptible bird class is closed to migration immediately the disease breaks out. This is in line with the measures taken to reduce the spread of the disease though Yusuf and Kosun [10] assumed that migration is allowed into the susceptible bird population. The model is formulated as a system of first order differential equations.

The disease-free steady state was analyzed and it was found that it will be stable only if the growth (birth) rate of birds $\left(\alpha_{\mathrm{B}}\right)$ has a bound. This means that if effort is made to reduce the growth rate of the birds immediately the disease breaks out, the disease will die out after enough time has passed and then we achieve a disease (flu)-free situation. That is, the disease-free state is asymptotically stable.

The endemic flu steady state was also considered. It was shown that the endemic flu steady state will be asymptotically stable if there is a bound on the infection transmission rate from birds to birds $\left(\beta_{\mathrm{B}}\right)$. This means that the disease will persist if the infection transition rate from birds to birds has a bound. This simply means that the disease will be endemic among humans.

From this analysis, we recommend that health officers and the Government should make effort to make sure that there is no migration of birds (chickens) from place to place immediately the disease breaks out. Effects should also be made to reduce the growth rate of birds (chickens) to bring about disease-free state. Chickens in poultry firms should all be killed immediately the disease is dictated and the owners should be compensated. This will help to reduce the spread and bring about a disease-free situation.

\section{REFERENCES}

Andrei, K., 2006. Lyapunov function and global stability of SIR and SIRS epidemiological models with nonlinear transmission.

Baym, M., 2005. Birds flu and you: An introduction to Mathematical Epidemiology. A seminar paper presented at SPAMS. Available in the net.

Beltrami, E., 1995. Mathematics for Dynamic Modeling Academic Press, Inc., Harcourt Brace Jovanovich Publishers, New York, P.17- 25.

Bowan, C. et al. 2005. A mathematical model for assessing control strategies against West Nile virus. Bulletin of Mathematical Biology 67: 1107-1133.

Ferguson, N.M., et al. 2006. Computer model of bird flu, Advance on line issue of Nature.

Longini, I, et al. 2004. Mathematical model of flu outbreaks. The New England Journal of Medicine, 351: 2363-2365.

Lewis, M., 2006. Traveling waves and spread rates for a West Nile virus model. Bulletin of Mathematical Biology.

World Health organization 2005. Epidemic and Pandemic Alert and Response.

World Health organization 2005. Report of the meeting on avian influenza and pandemic human influenza.

Yuauf. T. T. and Okosun, K.O., 2006. Mathematical model for bird flu disease transmission. Journal of the Nigerian Association of Mathematical Physics, 10: 465-470. 